\title{
Nanoética y nanomedicina. Apuntes para una nueva ética de la medicina
}

\author{
Fernando Sancén Contreras*
}

Parece novedoso hablar de nanoética y de nanomedicina. No lo es, sin embargo, hablar de ética, o de medicina, a secas. También es común hablar de la ética referida a la medicina. Al añadir el prefijo nano a la ética y a la medicina, queremos expresar su necesaria vinculación con la más reciente rama de la innovación científica y tecnológica. Nos referimos a la manipulación de la materia a nivel atómico, lo cual está generando una nueva forma de explicar la realidad, y una nueva forma de manejar los fenómenos físicos y vivientes. Nos referimos a las nanociencias y a las nanotecnologías (NyN). Actualmente, éstas expresan, en general, el estudio y la manipulación de la materia a escala nanométrica.

La importancia del trabajo científico y tecnológico a esta escala consiste en que en ella cambian las propiedades normales de los materiales físicos elementales, lo cual hace posible obtener nuevos productos con características y propiedades diferentes que se traducen en ahorro de energía, ahorro de materias primas, nuevos materiales, y nuevos insumos industriales, entre otras cosas. En el campo de la medicina, las NyN han aportado recientemente nuevas formas de administrar y construir fármacos, más eficientes equipos de diagnóstico y, en general, nuevas formas de influir en el cuidado de la salud de los individuos.

Por otra parte, desde una perspectiva ética, las NyN han desatado nuevos aspectos a considerar derivados de la obtención de nuevos materiales curativos y de nuevos aparatos que están modificando la vida de los seres humanos y los valores que a ella han sido vinculados. Así, por ejemplo, se plantea con urgencia el problema de precisar el ámbito terapéutico de lo que corresponde al mejoramiento o potenciación de las facultades del ser humano, especialmente en lo que se refiere a las actividades del cerebro. Las investigaciones neuronales, potenciadas por el trabajo a nanoescala están favoreciendo la construcción de dispositivos que permitirán dirigir las operaciones mentales de los individuos. ¿Dónde ubicar el límite, se cuestiona, entre la recuperación de las actividades normales interrumpidas por alguna enfermedad, y el mejoramiento de dichas facultades sin que hayan sido menguadas?

Esto, entre otros aspectos del reciente desarrollo de la ciencia y la tecnología, específicamente de las $\mathrm{NyN}$, ha hecho patente la necesidad de revisar el concepto mismo de ética, y el carácter absoluto que generalmente se atribuye a las normas que pretenden orientar las actividades de los individuos. En efecto, muchos de los adelantos recientes de los cuales disfrutamos nos ponen en contextos nuevos que requieren una mayor profundización y quizá de una nueva forma de considerar a la ética misma.

\footnotetext{
* Profesor en el Departamento de Política y Cultura de la Universidad Autónoma Metropolitana - Xochimilco. Email: fsancen@correo.xoc.uam.mx
} 
En este breve trabajo pretendemos presentar el terreno - nuevo sin duda-que se abre en la ciencia y tecnología actuales para la medicina con los aportes de las NyN; hablaremos también de los retos que plantean las NyN en el terreno de la acción humana tanto a los médicos e investigadores, como a la sociedad en general. Desde esta perspectiva la investigación y el desarrollo (I+D) que realizan la ciencia y la tecnología forman un campo prioritario para el trabajo de la ética, en la medida en que constituyen uno de los motores más poderosos del cambio para la humanidad en los tiempos actuales. Vale la pena, por tanto, preguntarse hacia dónde dirigir a la I+D para obtener los satisfactores que requiere la humanidad y lograr así una existencia plena para cada individuo. Postulamos abandonar aquella forma de ver a la ciencia y a la tecnología como neutras y sujetas a la reflexión ética sólo después de que ya estaban sus resultados circulando por la sociedad. Proponemos la conveniencia de construir una ética que vaya antes de la I+D para que les sirva de guía, y no como una simple reflexión acerca de la manera en que los resultados de la ciencia y la tecnología deben ser utilizados. Las NyN ofrecen la oportunidad, por su actual trascendencia respecto de la sociedad, para iniciar esta labor que se antoja importante y trascendente. Los límites de este trabajo sólo nos permitirán avanzar algunas ideas que seguramente cuestionarán otras posiciones y llevarán a un análisis más cuidadoso de la relación entre la sociedad actual y el desarrollo de las tecnologías emergentes.

\section{LAS NANOCIENCIAS Y LAS NANOTECNOLOGÍAS}

Por su reciente actualidad, consideramos útil para nuestro propósito precisar el concepto y alcances de las NyN. Se utiliza el prefijo nano para hacer referencia a una medida: el nanómetro, que equivale a una mil millonésima parte de un metro, es decir, $10^{-9}$ de un metro. Para tener una idea de estas dimensiones, consideremos que un cabello humano tiene un diámetro de alrededor de 80,000 nanómetros, y que un hilo de DNA mide alrededor de 2 nanómetros de ancho. Se dice, por otra parte, que la escala nanométrica se ubica en el rango comprendido entre uno y cien nanómetros; es decir, que los científicos al trabajar en las NyN lo hacen en dicho rango.

La importancia del trabajo a escala nanométrica consiste en que en esas dimensiones es posible controlar o cambiar las características fundamentales de un material cualquiera, incluyendo su punto de fusión, sus propiedades magnéticas, su resistencia, su conductividad, su porosidad, corrosividad, color, etc., y -lo más importante en algunos casos para la medicina- su biocompatibilidad, y todo esto sin cambiar la composición química del material con el que se trabaja a dicha escala.

En relación con lo anterior, se habla de nanociencia para expresar la rama de estudio acerca de las propiedades de los materiales y de su manipulación a escala atómica, molecular y macromolecular, donde las propiedades difieren significativamente de aquellas que tienen en la escala macro. En consecuencia, la nanociencia es el estudio y manipulación de la materia a escala nanométrica. ${ }^{1}$

\footnotetext{
En este trabajo asumimos la definición ofrecida en primera instancia por la Real Sociedad y la Academia de Ingeniería en julio del 2004, utilizada por el Grupo Europeo sobre Ética en la Ciencia y las Nuevas Tecnologías de la Comisión Europea. Ver "The European Group on Ethics in Science and New Technologies to the European Commision", Opinion on the ethical aspects of nanomedicine, Opinión N21, 17 de enero del 2007. A lo largo de este trabajo nos servimos de los conceptos ahí expuestos.
} 
El término nanotecnología se utiliza para significar el desarrollo y la aplicación práctica de estructuras y dispositivos a escala nanométrica en diversos campos, incluida la medicina. La nanotecnología se propone explotar en los diferentes materiales sus propiedades físicas, químicas y biológicas, las cuales, con frecuencia, difieren de las que se conocen en los tamaños a los cuales estamos acostumbrados.

Hoy las NyN están desarrollando innovaciones especialmente en tres campos, que interactúan permanentemente entre sí debido a la escala en que trabajan:

- Controles nuevos y mejorados con los que se mide y manipula a la materia en la escala nanométrica. Con estos controles es posible construir bloques y nanoaparatos.

- Caracterización nueva y mejorada de materiales a escala nanométrica.

- Nueva y mejor comprensión de las relaciones entre nanoestructuras y sus propiedades en vistas a conseguir su manipulación de manera eficiente.

Por su parte, la nanomedicina denota a la ciencia y a la tecnología que se ocupan de dignosticar, tratar y prevenir enfermedades y lesiones traumáticas mediante la aplicación de los resultados obtenidos en las NyN. Con los dispositivos, estructuras, medicamentos, compuestos, etc., utilizándolos como herramientas moleculares, la nanomedicina busca aliviar el dolor o preservar y mejorar la salud humana. También constituye un campo abierto al conocimiento más amplio de la dimensión molecular del ser humano. Generalmente, se considera que la nanomedicina abarca cinco subdisciplinas principales que en numerosas ocasiones se entrecruzan y sobreponen: a) herramientas de análisis; b) nano-imagen; c) nanomateriales y nanodispositivos; (d) nuevos sistemas terapéuticos y suministro de medicamentos y, e) los aspectos clínicos, regulatorios y toxicológicos. Con todo este potencial cuyo poder de velocidad y de precisión aumenta con las $\mathrm{NyN}$, la nanomedicina se yergue como un factor que cambiará la práctica de la medicina acarreando cambios significativos en la concepción misma de la medicina y en su aplicación para el mejoramiento de la existencia del ser humano. Problemas como la distinción entre curación y mejoramiento (enhancement), el acceso de toda la población a los nuevos tratamientos quirúrgicos, la manipulación de la información genética no sólo para la construcción de nuevos seres humanos, sino para la obtención de tejidos en el mismo organismo con propiedades diversas, traen necesariamente a discusión el problema de la ética para la práctica de la medicina, ahora con las nuevas potencialidades obtenidas por las NyN. Todo esto abre un campo de reflexión originado en la medicina, pero que toca aspectos universales del ser humano y que tradicionalmente se trata en el ámbito de la ética. Ahora bien, dado que estos cambios provienen de las NyN, empieza a ser utilizado el término de 'nanoética' para referirse a los problemas derivados de la incorporación en la sociedad de los resultados obtenidos por las NyN.

\section{La NANOMEdicina}

De acuerdo con lo anterior, y conforme al campo donde se practica la medicina humana, es posible conformar un campo de significado propio, la nanomedicina, el cual incorpora los aportes de las nanotecnologías al menos en los rubros que señalamos a continuación. 
Técnicas de diagnóstico: las actuales pruebas de diagnóstico basadas en nanodispositivos están haciendo que el diagnóstico sea realizado en la etapa presintomática de cualquier posible enfermedad. Esto permitirá la introducción de medidas terapéuticas previas sin necesidad de utilizar métodos curativos o de extracción, tal como sucede en la actualidad. En efecto, los sistemas de diagnóstico basados en las nanotecnologías tendrán mayor eficiencia en términos de resolución, sensitividad, especificidad, confiabilidad, reproducibilidad e integración, además de su disposición casi inmediata y permanente. Estas técnicas de diagnóstico ya están disponibles. ${ }^{2}$ En un futuro, quizás aún distante, los dispositivos miniaturizados y biocompatibles de los que se ocupan las NyN permanecerán en el cuerpo para monitorear y reportar permanentemente determinadas enfermedades como el cáncer, o enfermedades cardiovasculares, entre otras.

Imagen: las nuevas técnicas de diagnóstico basadas en las nanotecnologías que utilizan la imagenología proporcionan información más precisa que las actuales vinculadas con la imagen del organismo para detectar enfermedades. En efecto, la imagenología derivada de la nanotecnología ofrece mejores resultados que la tomografía, la resonancia magnética, o el ultrasonido, etc., actualmente en uso. Se sabe, por ejemplo, que los óxidos de acero súper-paramagnéticos con un diámetro menor a 5 nanómetros, permite obtener imágenes nítidas su utilización ha sido exitosa para detectar metástasis en su etapa inicial.

Biomateriales: las nanotecnologías aplicadas a los biomateriales mejoran los resultados actuales obtenidos con base en aleaciones metálicas, cerámicas, polímeros, y compuestos. Los nanobiomateriales permiten comprender e imitar las estructuras y rutas bioquímicas que guían a la cicatrización natural, para inducir y apoyar la cicatrización más allá de las capacidades normales. Se trabaja también para obtener mejores propiedades mecánicas y compatibilidad biológica en las prótesis e implantes dentales, catéteres, vendajes, y en todo el instrumental médico.

Desarrollo y suministro de fármacos: el suministro de fármacos es y será una de las más importantes aplicaciones de las nanotecnologías a la medicina. Los fármacos incorporados a nanotransportadores permiten mejorar su especificidad y su eficacia. Los nanofármacos se caracterizan por su complejidad, y pueden hacer llegar moléculas curativas específicamente al sitio donde se requieren atravesando las barreras biológicas que todo organismo posee. Al mismo tiempo, pueden contener reactivos específicos, como anticuerpos que alcanzan objetivos predefinidos como moléculas cancerígenas o células inflamadas y, una vez alcanzado su objetivo, se desintegrarán ahí mismo. Esto hará posible también otras formas de terapia, como la termoterapia que consiste en dotar de dispositivos a las nanopartículas para que ellas mismas se alojen en células malignas y luego sean calentadas localmente mediante radiaciones dirigidas especialmente a esos dispositivos para obtener efectos particulares en la quimioterapia o la radiación.

Medicina regenerativa: además de las terapias antes y después del diagnóstico que actualmente se desarrollan en las $\mathrm{NyN}$, se trabaja en la medicina regenerativa mediante la activación de genes que estimulan la regeneración de tejidos, con la producción de nanomembranas biocompatibles; mediante el mejoramiento en el funcionamiento y duración de prótesis neuronales, y mediante la creación de un nuevo linfocito que

\footnotetext{
2 Ver "The European Group on Ethics in Science and New Technologies to the European Commision", Opinion on the ethical aspects of nanomedicine, Opinión $\mathrm{N}^{\circ} 21,17$ de enero del 2007: 17.
} 
restablece la respuesta inmune normal en el paciente. Además de esto, la manipulación de las relaciones biológicas a escala nanométrica incrementará sensiblemente la funcionalidad y longevidad de tejidos implantados.

También las nanotecnologías se están desarrollando en el campo de la terapia con células madre y esto ofrece posibilidades alentadoras para la regeneración de tejidos dañados.

Una aplicación nanotecnológica en el caso de los implantes es la utilización de nanodispositivos electrónicos. Específicamente en el campo de la visión, se están utilizando dichos dispositivos en el ojo en forma de nanovideocámara cuyas señales serán procesadas por una microcomputadora que a su vez transmitirá la señal a una red de electrodos ubicados en el ojo. Con esto se logrará restaurar la visión en los invidentes. Lo mismo se está desarrollando para el caso de la audición.

Aplicaciones cosméticas: aunque las aplicaciones de la nanotecnología en los cosméticos puede no ser considerada como parte de la nanomedicina, es importante señalar que los avances logrados por la nanotecnología en esta rama tienen aplicaciones en la terapia de la piel. Muchos de estos productos están ya en el mercado y su crecimiento se estima en un $10 \%$ anual.

Nanomedicina y toxicología: la medicina no es la única forma en que se utilizan los avances obtenidos por las NyN. Sus resultados se emplean en una amplia gama de aplicaciones que van desde la industria automotriz hasta los textiles y cosméticos, pasando por los aparatos de la electrónica, con frecuencia utilizados para diferentes diagnósticos y mediciones. Aunque la mayor parte de las aplicaciones de las NyN no se ubican en la medicina, el uso de los nanomateriales puede traer consecuencias a la salud si se dispersan en el entorno. En estudios realizados en animales para detectar la presencia y la fuerza de las nanopartículas en células, se ha demostrado la presencia de algunos efectos tóxicos derivados de la presencia de nanopartículas en las células. Se sabe también que los efectos de las nanopartículas en el cuerpo humano provenientes del entorno, pueden tener efectos diferentes a las partículas normales que se encuentran en el medio ambiente. En consecuencia, las repercusiones tóxicas de la nanotecnología proyectan la necesidad de ser contemplada como parte de la nanomedicina, y dado que esto entraña la responsabilidad para cuidar, normar y desarrollar condiciones que la controlen, la toxicología es un punto de unión de la nanomedicina con la ética.

Todos estos rubros forman parte de la nanomedicina, que a su vez conforma una novedad constante en el trabajo de atención a la salud de los seres humanos. Sin embargo, la nanomedicina, sin bien es ya una realidad, es aún incipiente. Su desarrollo se puede ubicar en periodos de tiempo que van desde lo ya logrado y que está en uso, hasta las previsiones más difíciles siquiera de imaginar, pero en cuya dirección ya se está trabajando. Así, se habla comúnmente de las siguientes etapas del desarrollo de la nanomedicina:

1) Hasta el año 2000, se ubica la primera generación de productos que consiste en nanoestructuras pasivas, tales como:

- Nanoestructuras dispersas y de contacto, como aerosoles, coloides.

- Productos que incorporan nanoestructuras compuestas; metales nanoestructurados, polímeros, cerámicas, como revestimientos y nanopartículas reforzadas. 
2) Hasta el año 2005, nanoestructuras activas:

- Nanoestructuras bioactivas, con efectos en la salud, como medicamentos dirigidos y biodispositivos.

- Estructuras físicoquímicas activas y adaptativas, como transistores en 3D, amplificadores.

3) Para el año 2010 se espera la obtención de sistemas de nanosistemas, como montajes guiados, redes tridimensionales y nuevas arquitecturas jerárquicas, robótica y biosistemas evolutivos.

4) Para los años 2015 a 2020 será posible contar con nanosistemas moleculares, como dispositivos moleculares 'por diseño', diseño atómico, funciones emergentes.

El diagrama siguiente ilustra lo anterior:

Desarrollo de la nanomedicina

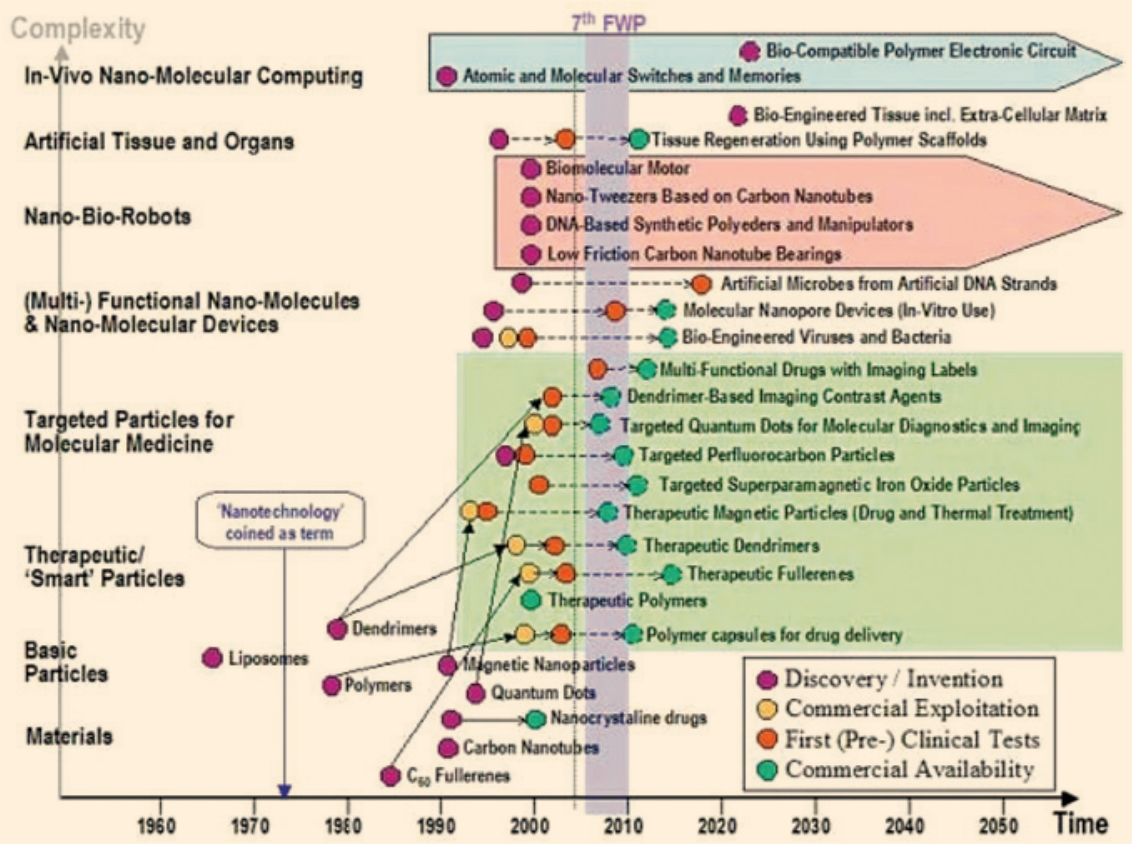

Fuente: Philips Medical Systems (7th FWP refers to the European Union's Seventh Framework Program) en la página <www.nanowerk.com/spotlight/id3938.jpg>. Consultada el 01/01/2009.

Con lo dicho hasta ahora contamos ya con la información más relevante acerca de la naturaleza y características actuales de la nanomedicina. Su importancia consiste en el alcance y desarrollo futuro que traerán cambios determinantes en la práctica médica y, en general en la vida cotidiana de los seres humanos. Esta influencia determinante de las nanociencias y las nanotecnologías en la medicina plantea preguntas 
pertinentes acerca de la dimensión ética de la práctica médica. El cambio que se está realizando, y los cambios que esperamos en el futuro próximo y remoto, influirán determinante y necesariamente no sólo en la forma de vida y la salud de los individuos, sino también cambiarán la actual forma de organización social, la manera en que cada individuo desempeña su propio trabajo, los valores vinculados con toda la vida desde su concepto mismo, hasta la forma de vivirla y la longevidad; afectará necesariamente las costumbres de los individuos, los valores sociales, y también el entorno biofísico, especialmente por la forma en que se estará trabajando sobre la materia y sobre las entidades vivientes, desde las células, hasta los más complejos organismos, como el cerebro humano. Las NyN y, concretamente, la nanomedicina plantean, en consecuencia, problemas a la concepción tradiciona de la ética, es decir, a la manera de comprender y de normar la acción individual, la forma de organización social que se asegura a sí misma a través de normas, en la medida en que las tecnologías emergentes están introduciendo cambios radicales que determinan dicha acción y organización.

\section{ÉTICA Y NANOMEDICINA}

A la ética se la relaciona comúnmente con la moral, es decir, con la determinación de lo que es bueno o malo. Generalmente se piensa acerca de la ética en términos de una norma o regla que permite medir la bondad o no de un determinado acto humano. Veremos que esta forma de ver la ética está cambiando en función de los cambios sociales derivados en gran medida por los avances de la CyT.

De manera más técnica, la ética, también llamada filosofía moral, es la disciplina que se ocupa del estudio de la moralidad; ésta constituye su materia de estudio u objeto. Se ocupa de los temas fundamentales acerca de la toma de decisiones para actuar, sobre todo del valor y de las normas desde las que se juzga que una acción es buena o mala. En consecuencia, la ética se refiere a todo sistema o teoría de valores morales o principios que guían a la acción humana, valores que son creación de los individuos para beneficiarse de su convivencia social.

Considerada como una rama de la filosofía, se vincula a la ética con otros campos de la acción del hombre, tales como la antropología, la economía, la política, o la sociología. Pero de manera más abstracta o profunda, la ética encuentra su explicación última en la ontología y la epistemología, dado que toda acción humana, cuyo estudio es lo propio de la ética, versa sobre el mundo, el cual requiere ser explicado (ontología), y conocido (epistemología). Generalmente se la divide en tres principales ramas: la metaética, la ética normativa, y la ética aplicada.

El estudio de los problemas vinculados con la naturaleza de los conceptos o juicios morales se considera que pertenece a la metaética. Esta disciplina pretende dar respuestas a preguntas tales como si un juicio moral es objetivo, es decir, independiente del sujeto que actúa, o subjetivo; si es absoluto o relativo.

La ética normativa se ocupa sobre todo del establecimiento de normas para la conducta humana, y se la asocia comúnmente con teorías generales sobre cómo se debe llevar la vida, siempre en un contexto cultural determinado. Una de las cuestiones centrales de la ética normativa es aquella que busca definir si la acción humana es buena o mala utilizando como criterio para ello las consecuencias derivadas de dicha acción. La ética normativa ve las normas, y la ética como teleológica o deontológica, es decir, en función de su conformidad con una norma o principio al que se le atribuye un valor absoluto. En esta perspectiva podría ser ubicada la filosofía moral de I. Kant. 
La ética aplicada, como su nombre lo dice, consiste en la aplicación de teorías normativas a problemas prácticos como igualdad racial y sexual, los derechos humanos, la justicia, la ecología, y aquellos problemas relacionados con el valor de la vida humana, que se hacen presentes, por ejemplo, en el análisis de la conveniencia de legalizar el aborto, o en lo relativo a la eutanasia. En relación con esta forma de considerar la ética, tenemos implicaciones éticas también en la medicina y en las ciencias biológicas, tales como la fecundación in vitro, el banco de espermas, la utilización de las células madre, la manipulación genética, entre otros.

Más allá de la clasificación de la ética, queremos avanzar algunas reflexiones sobre la ética en general, y su vinculación con la medicina. La relación de la medicina con la ética cobra hoy nuevas características, derivadas de la incorporación de las NyN a la investigación y práctica médicas, como lo señalamos. Aunque es común que al hablar de ética se piense en un conjunto de normas de validez universal casi absoluta que todo ser humano tiene la obligación de observar en el curso de su vida, una visión retrospectiva al pasado de la humanidad hace ver que las normas, algunas de las cuales hoy son consideradas inmutables y absolutas, no existían anteriormente. Tal es el caso, por ejemplo, de la libertad individual, que no se consideraba un valor absoluto vinculado a todo sujeto racional, puesto que antiguamente la esclavitud gozaba en aquellas antiguas sociedades de una justificación teórica y práctica. En culturas diversas, aún hoy, las normas que son válidas en una no lo son necesariamente en otra. Así, lo que consideramos como una norma absoluta e inmutable en nuestra sociedad, no lo es necesariamente en otra sociedad diferente en el tiempo y en el espacio. Por otra parte, en nuestra experiencia vemos que algunas instituciones y normas se han modificado en los últimos años como resultado de la introducción de nuevos aparatos, novedosos fármacos, etc., en nuestra vida diaria. Parecería, por tanto, que es necesario ver a la ética como el resultado de un conjunto de costumbres aceptadas por un determinado grupo social, y vinculadas con la necesidad de permanecer en la existencia mediante el intercambio constante con los miembros de dicho grupo social, y con su entorno físico y biológico. Esta forma de ver la ética cobra sentido y valor en el seno de la acción individual, es decir, en el ejercicio de la libertad de cada ser humano, y es investida de obligatoriedad en la medida en que mediante la observancia de las normas, derivadas de las costumbres, cada sujeto obtiene su propio bien en armonía con su entorno. En otras palabras, conforme a esta nueva visión de la ética, el quehacer humano no tiene otro objeto que el bien de quien actúa, con la certeza de que dicho bien proviene del entorno, hacia el cual obtiene, en consecuencia, una responsabilidad existencial, porque de éste depende la existencia, es decir, el bien de cada sujeto. De acuerdo con esto, podemos decir que la ética, además de analizar la pertinencia, validez y obligatoriedad de las normas en las condiciones históricas vigentes, se ocupa de la construcción del futuro del ser humano apuntando hacia nuevas formas de vida, a partir de su interacción con su propio entorno.

Esta breve descripción de la ética, tal como se presenta al hombre actual desde una perspectiva práctica, remite a la necesidad de dibujar una ética dinámica, impregnada de creatividad, en la medida en que toda acción humana es considerada como conformante y vuelta hacia el futuro de toda sociedad y de su entorno biofísico; se ve a la acción humana, y en consecuencia a su estudio, la ética, como responsable de dicho futuro que abarca al Universo mismo en la medida en que la existencia de la humanidad se desprende históricamente de dicho Universo. 
Desde esta perspectiva podemos contextualizar a la nanoética, que si bien comprende las normas derivadas de la filosofía moral en la medida en que incorpora en la práctica los principios fundamentales referidos a la existencia del ser humano, cuando la estudiamos ante la nueva realidad que nos presentan las NyN, surgen de inmediato cuestiones que sacuden la validez universal de dichas normas y obligan a la reflexión para asumir las nuevas cuestiones en el contexto del bien individual. Algunas de las cuestiones que las NyN plantean en el marco de la ética podrían formularse como sigue: ¿Cómo proteger el derecho fundamental a la salud de los ciudadanos cuando pueden ser expuestos a nanopartículas libres en el entorno? ¿Cómo promover el uso responsable de los dispositivos elaborados a escala nanométrica para proteger la vida humana y conducirla? ¿Cómo servirse de las NyN para contar con instituciones más justas? ¿Cómo conseguir con ellas superar la lacerante separación que se da entre los seres humanos; entre quienes tienen y aquellos que carecen prácticamente de todo? Estas preguntas llevan a analizar la manera de servirse de las NyN para la construcción de mejores condiciones de vida para el ser humano. La nanoética, por tanto, se refiere al futuro, aún inexistente, con la certeza de que la CyT son elementos de construcción de mejores condiciones para la existencia del hombre y el mejoramiento de su entorno. Al mencionar el futuro, nos situamos necesariamente en el tiempo. Pensar una ética vuelta hacia el futuro implica la incorporación del tiempo en su misma conceptualización, y éste lleva necesariamente al pasado, al presente, y al mismo futuro. En otras palabras, estamos ante una visión histórica tanto del hombre, como de las normas que éste se da para guiar su propia acción en el seno de la sociedad en la que transcurre su existencia.

La ética, en efecto, se nutre de la visión del hombre y del Universo en el que éste existe y se desenvuelve mediante sus acciones. En este sentido, podemos sostener que todas las acciones que cualquier individuo realiza están originadas y motivadas por el bien que considera obtener a través de su propia acción. El momento histórico de cada acción individual comprende todos los elementos que en ese momento se le presentan de manera consciente o bien inconscientemente. Ahí actúa su pasado como dato que en el momento presente ejerce un peso sobre su decisión; ahí actúa también la interpretación que realiza acerca de su situación actual, y actúa también el futuro hecho concreto en el bien que persigue; todo esto constituye un elemento actuante en la toma de decisión primero, y en la acción concreta después. Desde esta perspectiva que expresa un contexto dinámico, presentamos una visión de la ética vuelta hacia el futuro, y en tal sentido crítica, acerca de la existencia previa de normas absolutas, y propiciando un análisis constante de la validez del juicio moral que asume cada individuo en su actuar concreto. Presentamos una ética histórica, necesariamente vinculada con el futuro de cada individuo y también con el porvenir de su entorno tanto inmediato como mediato.

Esto, sin embargo, no puede ignorar los principios morales actualmente vigentes, en la medida en que ellos expresan el universo de validez que la sociedad se ha dado en determinado momento al considerar que con ellos cada individuo está en posibilidad de conseguir su propio bien.

Por otra parte, dichos principios morales se hacen concretos en cualquier sociedad a través del conjunto de reglas, normas, costumbres, etc., que generalmente se organizan, modifican y estudian en disciplinas del saber humano; nos referimos al derecho, a la política, a la economía, a la psicología, entre otras.

Por lo anterior, el análisis de la nanoética comprende todos estos campos que se desprenden del reconocimiento del individuo como el centro de toda reflexión ética. 
En efecto, del valor central que representa la existencia de cada individuo derivan sus derechos acerca de la dignidad humana, derechos que se expresan como protección, también llamados negativos en la medida en que expresan negaciones o limitaciones, pero también aquellos derechos, los positivos, que orientan hacia nuevas realidades.

Llevada al terreno de la nanomedicina, la nanoética se centra primero en la preservación de la dignidad e integridad del ser humano a través de la reflexión sociológica, la política, la economía y la antropología. Todos estos aspectos, si bien se tratan con frecuencia de manera aislada, en la realidad constituyen un todo interrelacionado, sobre todo cuando se habla de la nanomedicina. Ésta, desde una perspectiva ética, ha de ocuparse, en general, de que sean protegidos los derechos y aspiraciones del ser humano en la atención de su salud. De esta forma, la seguridad de los individuos constituye un punto central para la nanomedicina, y su contraparte, el riesgo del uso de nanomateriales en la medicina o fuera de ella es un tema propio de la nanomedicina. La valoración del riesgo se concibe como un factor vinculado con la protección de la dignidad humana de las personas y su autonomía, involucrada directamente a través de las aplicaciones médicas, o indirectamente por la posible exposición de las personas a las nanopartículas libres.

Para esto, se acepta comúnmente el llamado "principio precautorio", por el que se guía la práctica de la nanomedicina, y en general el uso de las nanotecnologías. En la práctica, el principio precautorio se aplica ahí donde una preliminar evaluación científicamente objetiva indica que existen fundamentos razonables para ver que los efectos potencialmente peligrosos para el medio ambiente, la salud humana, animal o de plantas, pueden ser inconsistentes con el nivel de protección elegido para la Comunidad. ${ }^{3}$

La relación entre nanoética y nanomedicina se nutre de los aspectos de la ética aplicados a la medicina. Algunos de ellos pueden señalarse de esta manera:

a) Derivado del valor fundamental que constituye la existencia de cada sujeto racional, la ética, en la práctica médica, asume la forma de protección de cada individuo. Esto se traduce en la obligación de proporcionar la información médica a la sociedad acerca del frecuente vacío de conocimiento y la incertidumbre de los resultados que se esperan en la atención médica a cada individuo. De la misma manera, la información médica predictiva, basada en los actuales desarrollos tecnológicos, constituye una obligación derivada de la protección hacia el individuo, a la que estamos todos obligados, especialmente las personas vinculadas con la atención a la salud.

b) El diagnóstico basado en los avances de la nanotecnología y el uso posterior de herramientas terapéuticas, también basadas en la nanotecnología, requieren necesariamente del consentimiento informado del paciente. Esto, a su vez, exige que se le proporcione una información comprensible para que valore adecuadamente los riesgos y la incertidumbre que contiene toda investigación médica general, y aquella que se realiza para cada paciente en particular.

c) Dado que la nanomedicina ofrece posibilidades de nuevos diagnósticos obtenidos con rapidez y precisión a nivel molecular, se da el caso de que no se

\footnotetext{
${ }^{3}$ Ver "The European Group on Ethics in Science and New Technologies to the European Commision", Opinion on the ethical aspects of nanomedicine, Opinión $\mathrm{N}^{\circ} 21,17$ de enero del 2007: 39.
} 
cuente todavía con la capacidad para hacer una adecuada interpretación de dichos diagnósticos y, más aún, que sean descubiertas nuevas enfermedades. Esta complejidad en el diagnóstico y la responsabilidad personal tanto del médico como del paciente son un campo propio para la decisión ética donde la información y la libertad son dos criterios fundamentales para asumir la responsabilidad ante la posible angustia derivada de las circunstancias mencionadas.

d) La información diagnóstica cada vez más rápida y precisa gracias a la nanotecnología, puede ser interceptada o proporcionada a terceros. Este riesgo constituye un terreno propio de la responsabilidad ética del personal de atención a la salud que obtiene los datos de aquellos equipos y procedimientos que son utilizados para elaborar el diagnóstico.

e) Otro rubro de la nanoética en la nanomedicina lo constituye la tenue distinción entre la utilización médica de químicos, fármacos y dispositivos, y la utilización para el mejoramiento del rendimiento físico y mental de las personas. No está suficientemente claro si un tratamiento determinado a una persona se considera como 'mejoramiento' (enhancement) o como tratamiento para superar un problema de salud. En todo caso, la precisión de esta distinción y su aplicación, caen dentro de la ética, específicamente de la nanoética, porque numerosos dispositivos están siendo pensados específicamente para mejorar el rendimiento de las facultades naturales de los individuos; tal es el caso de las neurociencias en la escala nanométrica.

f) Un gran reto para la ética en la nanomedicina, que no es exclusivo, lo constituye el posible acceso a las innovaciones derivadas de la nanomedicina debido a los costos que representan. Aunque todo individuo luchará por acceder a dichas innovaciones, habrá quien no pueda pagar su alto costo y quedará al margen, constituyendo seguramente un conjunto mayoritario de ciudadanos de segunda clase. También a nivel individual, la nanoética podrá precisar cómo los individuos lograrán disponer, o no, para su vida personal y familiar, los aportes determinantes de la nanomedicina una vez que cuenten con la información adecuada.

g) La ética se ve afectada por los avances de la nanomedicina también respecto a la responsabilidad de quienes trabajan en $\mathrm{I}+\mathrm{D}$ de todo lo relacionado con la atención a la salud con dispositivos a escala nanométrica; son los investigadores los primeros que deben tener conciencia de la importancia de su labor para obtener dispositivos adecuados al medio ambiente, controlando la toxicidad y adecuados para la salud de los individuos. Además, los grupos de investigación y el personal médico pueden verse afectados por las nanopartículas desprendidas de dispositivos y medicamentos nanotecnológicos bien sea por una afectación directa en su salud, o bien sea por hacerse inmunes a los nanomedicamentos. El trabajo en el laboratorio nanométrico y la aplicación de la nanomedicina para la atención a la salud cobran así una dimensión ética para cuidar de la integración y salud de dicho personal.

Aparte de estos aspectos que vinculan la práctica de la nanomedicina con la ética, existen los temas sociales para la ética, donde se plantean cuestionamientos en el campo de lo económico, lo social, lo político y, sobre todo, en lo que se refiere al gobierno y las instituciones vinculadas con la práctica y el análisis de los resultados de 
la nanomedicina. Estos aspectos no los tocamos en este trabajo, como tampoco tratamos el uso militar que puede darse a la nanomedicina, o lo referente a la propiedad intelectual de las innovaciones de las nanotecnologías llevadas al terreno de la medicina. Sin embargo, son aspectos fundamentales para el estudio de las NyN y sus implicaciones en la sociedad actual.

\section{ConcLusión}

De lo anterior podemos concluir que tanto la ética como la medicina se encuentran en un contexto totalmente innovador y dinámico conformado por los resultados obtenidos hasta hoy y los que seguramente se obtendrán en el futuro gracias al trabajo a nanoescala de las NyN.

La nonomedicina tiene un interés legítimo por aplicar dichos resultados para cuidar y reparar la salud humana. Por su parte, la nanoética propone desarrollar las aportaciones de las NyN imprimiéndoles una orientación fundamental que consiste en asumir como guía tanto de las instituciones que financian proyectos, como de los investigadores que los implementan, aquello que favorece la plena y digna existencia del hombre.

Este criterio o valor fundamental —el sujeto- deberá guiar toda innovación y toda investigación científica y tecnológica, especialmente aquella que está orientada al cuidado y mejoramiento del ser humano. Se trata de un criterio basado en el presente, pero orientado al futuro de la sociedad vista como el entorno que debe favorecer siempre el desarrollo pleno de cada uno de sus miembros. No está por demás citar que la sociedad de hoy es una sociedad globalizada, y que nos referimos al ser humano de cualquier latitud, cultura, religión o inclinaciones personales. En efecto, gracias a la interrelación global en que existe el hombre de hoy, cualquier acción individual cobra rasgos de universalidad. De ahí la trascendencia y actualidad de la reflexión ética desde una perspectiva histórica como hemos tratado de señalar. 